\section{Category}

Synthesis of Natural

Products and

Potential Drugs

Key words

( \pm )-paeoniflorigenin

Paeonia lactiflora

Prilezhaev reaction

Reformatsky-type cyclization

E. J. COREY*, Y.-J. WU (HARVARD UNIVERSITY, CAMBRIDGE, USA)

Total Synthesis of $( \pm)$-Paeoniflorigenin and Paeoniflorin

J. Am. Chem. Soc. 1993, 115, 8871-8872, DOI: 10.1021/ja00072a063.

\title{
Synthesis of $( \pm)$-Paeoniflorigenin
}

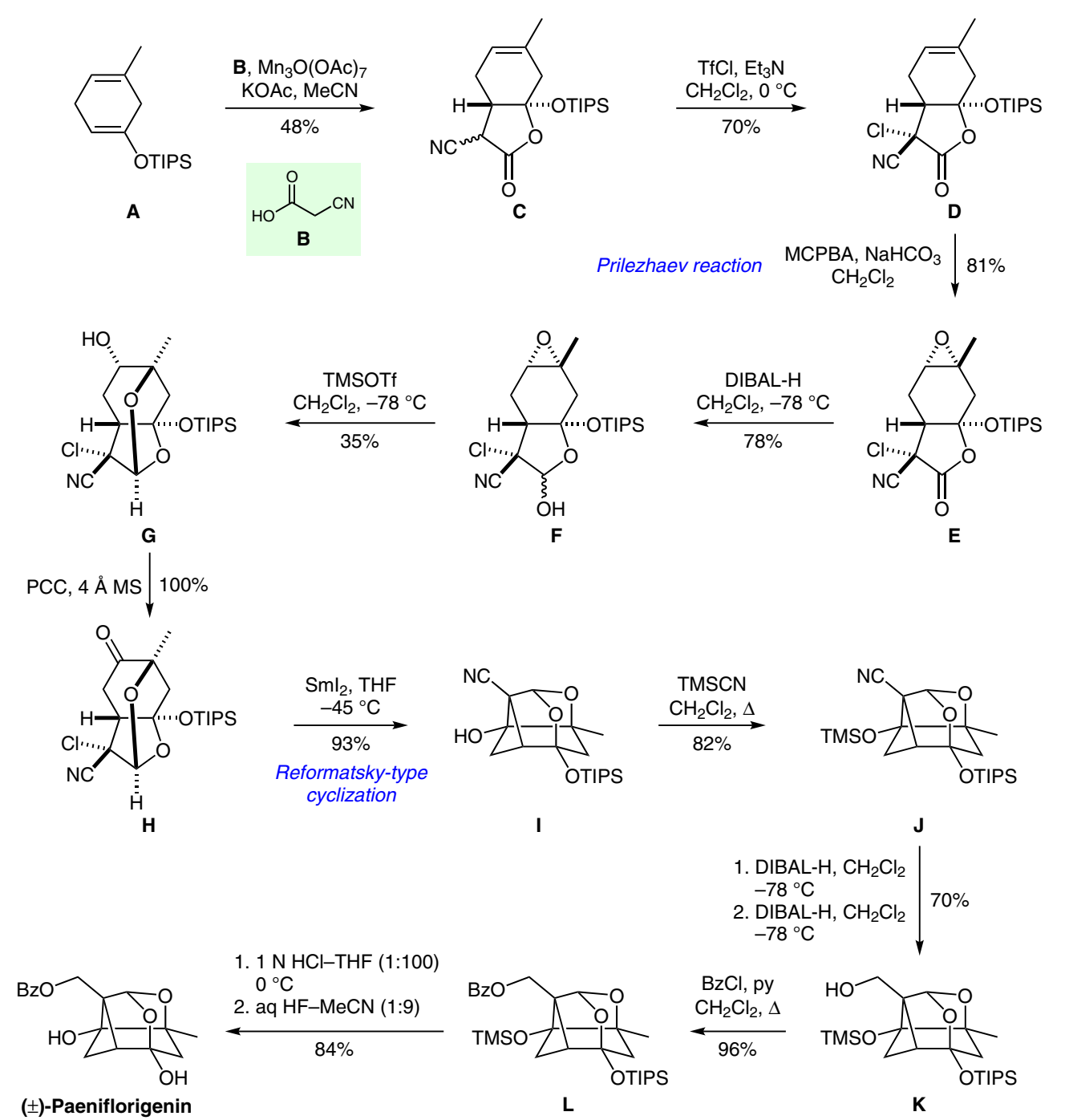

Significance: In 1993, Corey and Wu reported the synthesis of $( \pm)$-paeoniflorigenin, a natural product from the roots of paenia lactiflora, which are widely used in traditional Chinese medicine. The approach relies on oxidative annulation of dihydro-m-cresol ether $\mathbf{A}$ and $\mathrm{Sml}_{2}$-induced, Reformatsky-type cyclization from an $\alpha$-chloro nitrile.
Comment: The synthesis commenced with annulation of $\mathbf{A}$ and $\mathbf{B}$ to give $\boldsymbol{\gamma}$-lactone $\mathbf{C}$. Chlorination followed by Prilezhaev epoxidation yields E. Cyclization is achieved by treating lactol $\mathbf{F}$ with an excess of TMS-triflate. Exposure of $\mathbf{H}$ to $\mathrm{Sml}_{2}$ leads to formation of the carbon skeleton of paeniflorigenin. Protection of the secondary alcohol followed by reduction of the nitrile gives $\boldsymbol{K}$. Benzoylation and global deprotection completed the total synthesis of ( \pm )-paeniflorigenin in 13 steps from $\mathbf{A}$. 